Apidologie, 1985, 16 (3), 241-264

\title{
DIE HONIGBIENEN DES IRAN \\ 2. APIS MELLIFERA MEDA SKORIKOW, DIE PERSISCHE BIENE
}

\author{
F. RUTTNER *, D. POURASGHAR ** und D. KAUHAUSEN * \\ * Institut für Bienenkunde (Polytechnische Gesellschaft), \\ Universität Frankfurt, D-6370 Oberursel \\ *:* College of Agriculture, University of Tabriz, Iran
}

\section{ZUSAMMENFASSUNG}

Es wurden 63 Bienenproben aus dem Iran morphometrisch untersucht und unter Anwendung variationsstatistischer Methoden mit Proben aus umliegenden Ländern verglichen. Die südöstliche Begrenzung des Verbreitungsareals der Spezies A. mellifera wurde bestimmt. Die Bienen des Iran gehören zusammen mit den Bienen des irakischen Berglandes und SO Anatoliens zu einer einzigen geographischen Rasse, Apis mellifera meda Skokıкow (1929). Es ist eine sehr gelbe, mittelgroße Biene, die in vielen Merkmalen der Italienischen Biene A.m. ligustica ähnelt. Innerhalb dieser Rasse können morphometrisch sechs geographische Untergruppen festgestellt werden. Die geographische Variabilität innerhalb von A.m. meda zeigt andere Tendenzen, als sie z.B. in Afrika gefunden wurden. Die Stellung der Persischen Biene im Rahmen des feintaxonomischen Systems von A. mellifera wird diskutiert. Über ihr Verhalten gibt es nur Angaben aus bestimmten Regionen. Bezüglich des Schwarmverhaltens und der Verwendung von Kittharz ähnelt diese Biene mehr den kaukasisch-europäischen Rassen als den orientalischen.

\section{EINLEITUNG}

Im Gegensatz zur Zwerghonigbiene, $A$. florea, die nur in einem schmalen Küstenstreifen entlang des Persischen Golfes gefunden wird (RUTTNER et al., 1985), kommt $A$. mellifera in allen übrigen Gebieten des Landes vor, soweit ausreichende Vegetation vorhanden ist. Im Iran ist $A$. mellifera die Biene des Hochlandes und der Gebirge. Die größte Bienendichte wird im Westen, in Azarbaijan, erreicht. Aber auch in den Gebirgen von Alborz, Kordestan, Lorestan, Fars, Massandaran und Nord-Khorasan werden seit alters her Bienenvölker gehalten und es gibt hier, soferne Nistplätze vorhanden sind, auch wilde Bienenvölker. Die Zahl der Bienenvölker im Iran wird auf eine Million geschätzt, doch 
sind diese Angaben sicher ungenau, da sich noch immer etwa $2 / 3$ der Völker in traditionellen Behältern aus Ton, Holz oder Rutengeflecht befinden, die kaum vollständig erfaßbar sind (Tirgari, 1971; Pourasghar, 1979). Gegenwärtig (1985) wird die Zahl der Bienenvölker im Iran auf 1.6 Mill. geschätzt, von denen etwa $65 \%$ in Magazinen gehalten werden.

Die Verbreitungsgrenze von $A$. mellifera im Osten des Iran war bisher nur im nördlichen Abschnitt, im Gebiet von Mashad, bekannt. Nachdem SchNeIder und DJalal (1970) die westlichsten Völker von A. cerana in Zentral-Afghanistan aufgefunden hatten, stellte P. NoGgE, 1974, das nordöstlichste Vorkommen von $A$. mellifera im Iran in den Bergen NW von Mashad fest (Abb. 1). Über die Verbreitungsgrenze von $A$. mellifera in SO Iran war bisher nichts bekannt. Einen zweifelsfreien Nachweis von $A$. cerana im Iran gibt es bisher nicht.

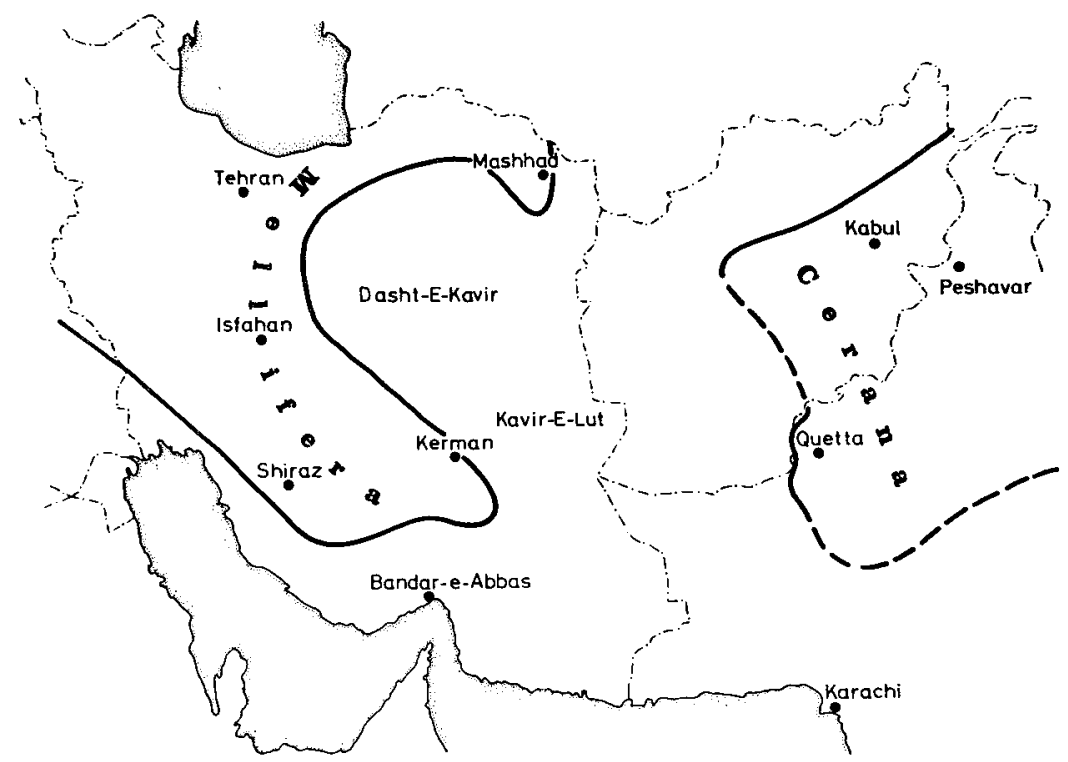

Aвв. 1. - Ostgrenze von Apis mellifera in Iran und Westgrenze von Apis cerana in Afghanistan und Pakistan

Fig. 1. - Eastern border of Apis mellifera in Iran and western border of Apis cerana in Afghanistan and Pakistan

Trotz einer sehr alten Imkertradition und einer in manchen Gebieten intensiven modernen Bienenzucht wurde bisher noch kein Versuch unternommen, die mellifera-Bienen des Iran $\mathrm{zu}$ beschreiben und taxonomisch $\mathrm{zu}$ klassifizieren. Lediglich von A.S. SKorikow (1929) gibt es eine Notiz über cine von der Armenischen Biene unterscheidbare «A.m. meda» mit Vorkommen «im Kreis Lenkoran und in Nordpersien». Außer einer Abbildung des 2. Sternits einer 
Arbeitsbiene, die eine «breite Vorderkante» zeigen soll, werden aber keine Angaben über diagnostisch verwertbare Merkmale gemacht. SKORIKOW gab dieser Biene den Status einer Spezies und ordnete ihr auch die Syrische und die Zyprische Biene zu. Gostze (1930) übernahm zunächst diese Klassifizierung, ohne allerdings später $(1940,1964)$ wieder darauf zurïckzukommen.

\section{MATERIAL UND METHODEN}

Seit vielen Jahren wurden methodisch Bienenproben aus dem Iran durch Veïmittlung mehrerer Fachkollegen aus diesem Lande gesammelt. Der Großteil der in dieser Studie analysierten Proben wurde jedoch von einem der Autoren (D. Pourasghar) auf ausgedehnten Reisen durch das Land zusammengetragen, wobei insgesamt etwa $45000 \mathrm{~km}$ zurückgelegt werden mußten. Dabei wurden vor allem entlegene Bienenstände mit traditionelfer Betriebsweise ausgewählt, da angesichts der viele Jahre währenden massiven Importe von Bienenköniginnen aus Australien und den USA in manchen Gebieten mit Hybriden (mit ligustica und carnica) zu rechnen war.

Zum Vergleich wurden Bienenproben aus den benachbarten Ländern Türkci und Iraq, sowie aus Zypern, Libanon, Israel und Jordanien in die Analyse einbezogen. Dicse Proben stammen zum Großteil aus der umfangreichen Sammlung von BRUDER ADAM, die uns freundlicherweise von ihm, bzw. vom Bee Department der Rothamsted Experimental Station zur Verfügung gestellt wurden. Die Proben aus Israel wurden zu einer Zeit gesammelt (1954), als dort noch nicht die Umstellung auf importierte Stämme erfolgt war.

Insgesamt standen für die Analyse 169 Bienenproben von (im Regelfall) je 20 Bienen zur Verfügung, verteilt auf folgende Länder :

$\begin{array}{lr}\text { Iran } & 63 \text { Proben } \\ \text { Türkei } & 63 \text { Proben } \\ \text { lrak } & 16 \text { Proben } \\ \text { Libanon, Israel, Jordanien } & 21 \text { Proben } \\ \text { Zypern } & 6 \text { Proben }\end{array}$

Bienen von der Arabischen Halbinsel (Saudi Arabien, Yemen und Oman) wurden nicht einbezogen, weil diese einem ganz anderem Sektor der mellifera-Rassen angehören (Äthiopische Region; RUTTNER, 1975). Aus den nördlich anschließenden Gebieten der UdSSR (Armenien, Georgien) stehen dzt. noch keine fertig analysierten Proben zur Verfügung. Für Analysen innerhalb des Verbreitungsgebietes von $A . m$. meda mußten einige Proben ausgesondert werden. werden, weil sie wegen Wanderung mit Sicherheit nicht als typisch für die Region gelten können (für einzelne andere Proben ist dies ebenfalls nicht auszuschließen). Deshalb wurde nicht für alle Analysen dieselbe Probenzahl verwendet.

Die Bienen wurden in heißem Wasser oder mit Dämpfen von Diäthyläther abgetötet und in Pampell-Gemisch aufbewahrt. Die morphometrische Bearbeitung erfolgte nach der von Rurrner et al. (1978) beschriebenen Standardmethode.

\section{Statistische Verfahren}

Die für die statistische Bearbeitung ausgewählten 34 Merkmale verteilen sich auf vier Gruppen (Größe, Behaarung, Flügelgeäder und Farbe), beschrieben bei RutTNER et al. (1978). In den Analysen wurden nur primäre Meßdaten verwendet. Für die Beschreibung der geographischen Gruppen erweisen sich aber auch abgeleitete Daten wie Summen (z.B. Gesamtlänge des Beines), Proportionen (z.B. Verhältnis von Körpergröße und Beinlänge) und Indices (Cubital-Index) als sehr informativ.

Als Grundeinheit (OTU) für die numerischen Analysenverfahren wurden nicht die Meßdaten 
der Eınzelbienen, sondern die normierten Mittelwerte jeder Bienenprobe (20 Bienen aus einem Volk) verwendet. Die Symbole in den graphischen Darstellungen repräsentieren also die «Standardbiene» einer Probe.

Zur Aufklärung der taxonomischen Struktur der Populationen aus den genannten Gebieten wurden zwei verschiedene multivariate Analysenverfahren angewandt :

- eine Faktorenanalyse (principal components analysis, p.c.a.)

Die graphische Darstellung der OTU's in Abhängigkeit von den errechneten Faktoren zeigt im Idealfall für jede morphometrisch unterscheidbare Population einen klar abgegrenzten «Cluster». Bei Populationen, die kontinuierlich ineinander übergehen, überlappen die Cluster; bei Stichproben aus einer einheitlichen Population würde totale Überlappung auftreten. Die Distanzen zwischen den Clustern repräsentieren die Ahnlichkeit der Gruppen in bezug auf die errechneten Faktoren und damit auf die enthaltenen Variablen. Daher müssen zwei Gruppen dann als trennbar gelten, wenn sie mindestens bezüglich eines Faktors keinen Überlappungsbereich aufweisen.

\section{- eine schrittweise Diskriminanzanalyse}

Hiermit werden vorgebene Gruppen (die in diesem Fall aus der Faktorenanalyse extrahiert wurden) auf ihre interne Variabilität im Vergleich zur Gesamtvariabilität geprüft. Bei der schrittweisen Methode werden alle Variablen nacheinander zur Analyse hinzugezogen, beginnend mit der Variablen, die am meisten zur Trennung der Gruppen beiträgt, usw. Durch Eliminierung der Variablen, die keinen Beitrag zur Trennung der Gruppen leisten, entsteht eine bessere Diskriminierung.

Für die Diskriminanzanalyse «Vorderer Orient» (Abb, 3) wurden 13 Variable gewählt (Merkmale der Größe $5 x$, des Flügelgeäders $6 x$, der Farbe und Behaarung je $1 \mathrm{x}$ ). Für die Diskriminanzanalyse «Meda intern» (Abb. 5, 6) genügten 9 Merkmale (Größe und Geäder je $4 x$, Behaarung $1 \mathrm{x}$ ).

Das Ergebnis der Diskriminanzanalyse sind wie bei der Faktorenanalyse unregelmäßige Cluster aus Einzelproben. Eine bessere Darstellung der Populationen erreicht man durch Errechnung von «Konfidenz-Ellipsen» (CoRnuet, 1982), deren Größe für jeden beliebigen F-Wert berechnet werden kann.

Die Bearbeitung des umfangreichen Datenmaterials erfolgte auf der Rechenanlage des Hochschulrechenzentrums der Universität Frankfurt am Main (UNIVAC Serie 1100), dic Konfidenzellipsen wurden auf einem HP-Rechner (Typ 7725) berechnet und auf einem angeschlossenen Plotter (Typ 7470 A) gezeichnet.

Verwendete Programme : BMDP Biomedical Computer Programs P-Series 1979. Ed. : W.J. Dixon, M.B. Brown, University of California Press.

\section{ERGEBNISSE}

\subsection{Verbreitung von Apis mellifera im Iran}

Im Südosten des Landes wurde das östlichste Vorkommen von A. mellifera in den Bergen nördlich von Sabzevaran, SO von Kerman, festgestellt. Aus der Art der traditionellen Bienenwohnungen (liegende Klotzbeuten, Abb. 8) und der isolierten Lage kann geschlossen werden, daß es sich um ein autochthones Vorkommen handelt.

Weiter östlich wurde nur noch $A$. florea gefunden, die in Baluchistan vor 
allem an der Küste (Chah Bahar), aber auch weiter im Inland (Bazman bei Iranshar) gefunden wurde.

Die östlichsten mellifera-Funde stammen im Norden von $59^{\circ}$ (Raum Mashad), im Süden von $58^{\circ}$ östlicher Länge. Das ist ungefähr die Linie des östlichsten autochthonen Vorkommens von A. mellifera weiter im Norden, im südlichen Ural (Alpatov, 1948).

Die zentralpersischen Wüsten Kavir und Lut sind vollständig bienenfrei. Damit gewinnt das Verbreitungsgebiet von Apis mellifera im Iran die Form einer nach Osten geöffneten Zange (Abb. 1).

Zur Westgrenze von $A$. cerana, der nächstverwandten Art, besteht überall eine Distanz von mehr als $500 \mathrm{~km}$.

Besondere Beachtung wurde der Frage eines natürlichen sympatrischen Vorkommens von $A$. mellifera mit $A$. florea geschenkt. Kontakte zwischen diesen beiden Arten wurden an zwei Stellen gefunden :

1. In Ahwaz, in der mesopotamischen Ebene, wurde A. florea erstmals für den Iran festgestellt (TIRGARI, 1971), gleichzeitig werden aber dort auch melliferaVölker in modernen Kästen gehalten, allerdings nur wintersüber. Nachfragen haben ergeben, daß es bis vor einigen Jahren mellifera-Völker dort nicht gegeben hat und daß die mesopotamische Ebene erst im Zuge der modernen großräumigen Wanderimkerei zum Winterquartier für Bienenvölker aus dem nördlichen Hochland wurde.

2. Nördlich von Bandar Abbas wandern florea-Kolonien während der Sommermonate von der heißen Küstenzone in die Randgebirge bis in $800 \mathrm{~m}$ Höhe, wo es schon mellifera-Völker gibt. Auf Dauer leben auch hier $A$. florea und $A$. mellifera nicht im selben Gebiet.

Als Ergebnis ist festzuhalten, daß im Iran kein permanentes sympatrisches Vorkommen von $A$. florea und $A$. mellifera beobachtet wurde.

\subsection{Taxonomische Einordnung der iranischen Apis mellifera}

Die Multivariantanalysen der 167 Bienenproben aus den oben genannten Ländern des Vorderen Orients ergibt bei Anwendung der p.c.a. vier Cluster. Die aus der Diskriminanzanalyse berechneten Cluster bleiben auch bei einer Konfidenzgrenze von $90 \%$ weitgehend getrennt (Abb. 2). Der am weitesten vom Zentrum des Diagramms entfernte Cluster enthält sämtliche Proben aus dem Libanon, aus Israel und Jordanien, entspricht also der altbekannten A.m. syriaca BUtTEL-REEPEN, 1906 ( $\ll \mathrm{S} \gg)$. 


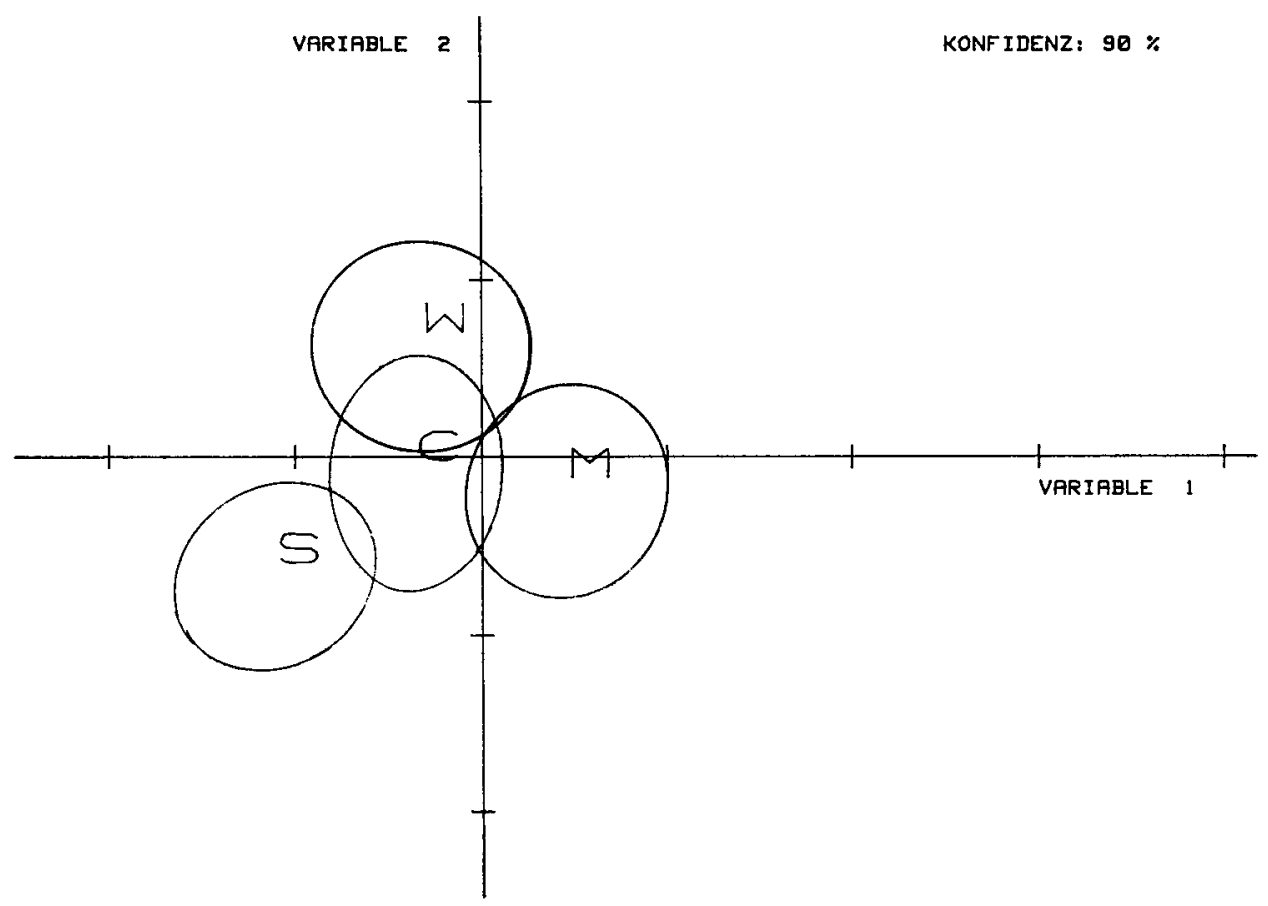

AвB. 2. - Diagramm nach den Daten einer Diskriminanzanalyse von Bienenproben aus dem Vorderen Orient

Vertrauensgrenzen $90 \% . \mathrm{N}=167$, Merkmale $=13$

FIG. 2. - Discriminant analysis of bee samples of the Near East

Limits of confidence $90 \% . \mathrm{N}=167$, characters $=13$

C. A.m. cypria, M A.m. meda, S A.m. syriaca, W A.m. anatoliaca

Eine zweite, große Ellipse (Abb. $2 \ll W »$ ) enthält 46 Proben aus Zentralund Westanatolien und aus der europäischen Türkei. Diese «Anatolische Biene » spielt im Zuchtprogramm von Bruder Adam eine große Rolle, wurde aber bisher morphometrisch noch nicht analysiert.

Eng an den Cluster der Anatolischen Biene angelehnt, ihn bei $75 \%$ Konfidenz beriihrend und bei $90 \%$ (ebenso wie die beiden übrigen Cluster) überlappend, ist der Cluster von A.m. cypria PollmanN, 1879 (« C»). Die graphische Präsentation einer Faktorenanalyse ergab für die beiden Rassen (Zyprische und Anatolische Biene) keine Trennung durch Faktor 1, aber Trennung anhand der Faktoren 2 und 3. Das weist darauf hin, daß die wichtigsten diskriminierenden Merkmale nicht solche der Größe sind (enthalten vor allem in Faktor 1), sondern des Flügelgeäders und der Behaarung. 
Die am weitesten rechts auf der horizontalen Achse liegende Ellipse umfaßt sämtliche 61 Proben aus dem Iran ( $M M \gg)$. Dazu kommen noch alle 16 Proben aus den Bergen des nördlichen Iraq und 16 Proben aus SO Anatolien, die alle zusammen eine geschlossene Gruppe bilden. Da auch die Proben aus dem äußersten NW, unmittelbar von der sowjetischen Grenze, derselben Gruppe angehören, muß die Iranische Biene als Apis mellifera meda SkoRIKow, 1929, bezeichnet werden.

Die Herkunftsregion der Proben aus dem Iraq, das nördliche Bergland, bildet das wichtigste Bienenzuchtgebiet des Landes, in dem auch Wildvölker vorkommen. Im Flachland (etwa in Baghdad) vorhandene Bienenvölker sollen alle aus den Bergen dorthin transportiert worden sein (KHALEL, 1955; G. Salman, pers. Mitteilung).

In Ostanatolien ist A.m. meda in dem Gebiet um den Van See und nach Westen bis Elacig - Tunceli und Malatya zu finden. Trotz des Fehlens geographischer Barrieren ist die morphometrische Abgrenzug gegenuiber der Anatolischen Biene sehr scharf. Im nordwestlichen Grenzbereich von Tunceli ist eine Probe (Nr. 15) morphometrisch eindeutig A.m. meda zuzuordnen, eine zweite (Nr. 16) ist der Anatolischen Biene angenähert. Die augenfälligsten Merkmale der untersuchten Rassen aus Vorderasien sind in Tab. 1 zusammengestellt. Meda nimmt im Vergleich zu den anderen Rassen hinsichtlich aller Merkmale eine mittlere Stellung ein : Anatoliaca ist größer und langhaariger als meda, cypria und syriaca kleiner und kurzhaariger. Unterschiede bestehen auch in den Körperproportionen. Meda ist, im Verhältnis zur Körpergröße, die kurzbeinigste dieser Rassen; hinsichtlich der relativen Flügellänge nimmt sie eine Mittelstellung ein. Die Proportionen des Flügels selbst (Breite/Länge) zeigen eine breite Form an. Dasselbe ist von der Form des Hinterleibs zu sagen (Schlankheits-Index, SI) : Meda ist breiter als die anderen Rassen des Vorderen Orients (und Afrikas).

Sehr deutliche Unterschiede ergeben sich auch bei den meisten der $13 \mathrm{er}$ faßten Merkmale des Flügelgeäders, von denen in Tab. 1 nur der Cubital-Index (CI) angeführt ist.

Merkmale der Farbe hingegen tragen nur wenig zur Diskriminanz der Bienenrassen dieser Region bei. Alle sind gelb, am Abdomen wie am Scutellum, mit nur geringer Variation (Klasse 7.7-8.0, bzw. 4.8-6.0). Eine Ausnahme bildet die Anatolische Biene, die durchwegs um zwei Klassenwerte dunkler ist.

Zur Einordnung von A.m. meda in das Schema des taxonomischen Systems der Spezies (RUTTNER et al., 1978) ist ein Vergleich mit A.m. ligustica von Interesse. Ligustica ist eine Rasse mittlerer Größe, in dieser Hinsicht zwischen der «großen» carnica (bzw. mellifera) und den südlicheren Rassen eingeordnet, und sie ist die am weitesten verbreitete und die bekannteste Bienenrasse überhaupt. 


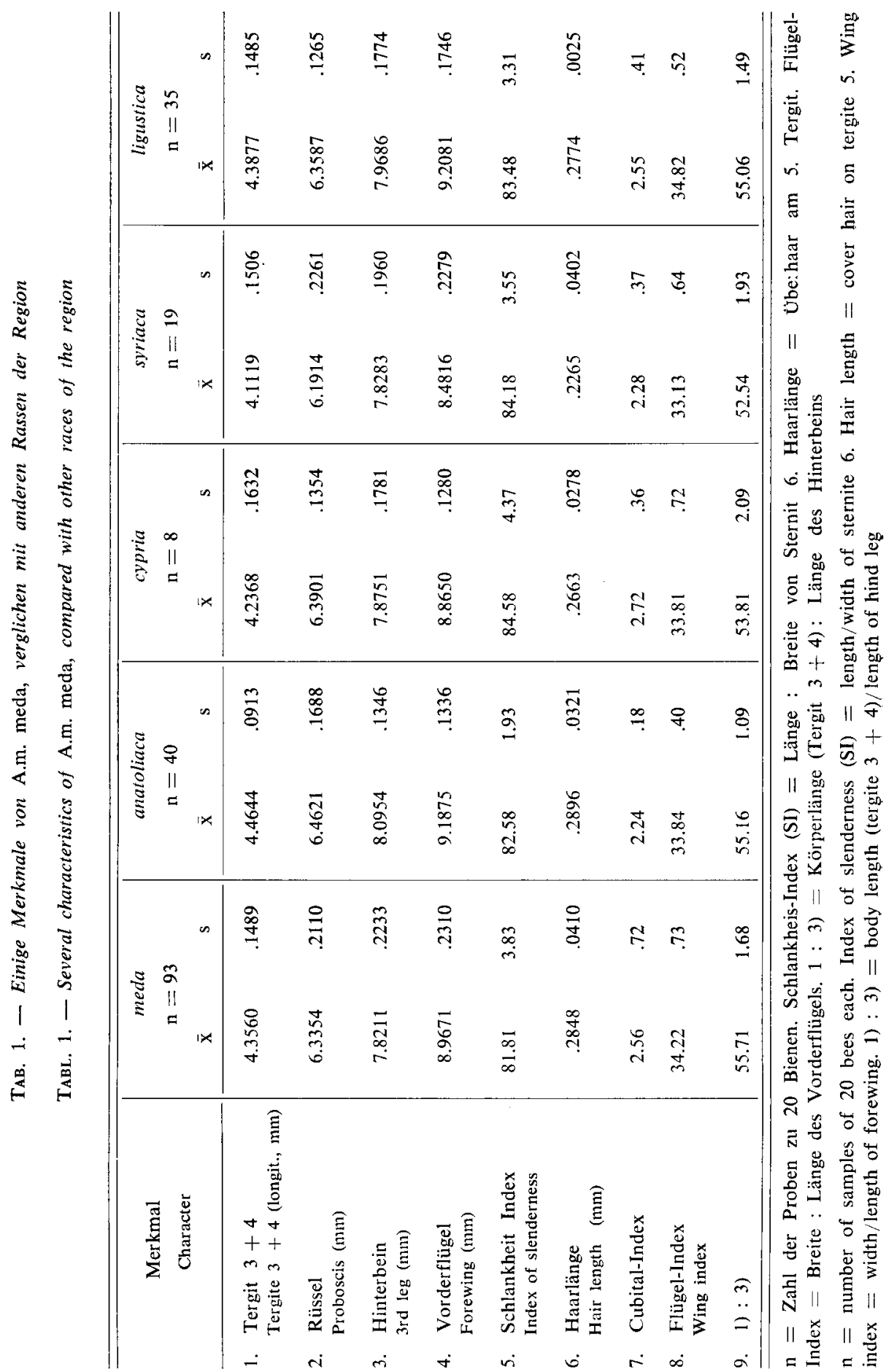


Tab. 1 zeigt eine erstaunliche Übereinstimmung in Rüssel- und Körperlänge, $\mathrm{CI}$ und der überdurchschnittlichen Flügelbreite (die bei ligustica noch stärker ausgeprägt ist). Aber ligustica hat längere Beine und Flügel, kürzere Haare und ein schlankeres Abdomen. In der Pigmentierung besteht bei den Abdominaltergiten kein Unterschied, hingegen ist das Scutellum bei meda deutlich gelber.

Wird in die p.c.a. des Vorderen Orients (34 Merkmale) ligustica mit einbezogen, so gehen diese Proben vollständig im Meda-Cluster auf. Eine ganz klare Trennung der beiden Rassen gelingt aber mittels der stufenweisen Diskriminanzanalyse, wobei zur kanonischen Trennung nur 5 Merkmale erforderlich sind (longitud. Durchmesser von Tergit 3, Breite des Vorderflügels, Haarlänge und die beiden Aderwinkel G 18 und I 10).

\subsection{Morphometrische Variabilität innerhalb von A. meda}

Das Verbreitungsgebiet von $A$. meda mißt etwa $2000 \mathrm{~km}$ von Ost nach West und ca $1000 \mathrm{~km}$ von Nord nach Süd. Es hat deshalb nahe gelegen, innerhalb dieses Gebietes nach morphometrisch faßbaren Unterschieden zu suchen. Diese Analyse mußte, um brauchbare Resultate zu liefern, auf autochthone Bienenvölker beschränkt werden. Es wurden deshalb alle Proben aus Teheran (reine Wanderbienenzucht mit vielen Importen) und aus Ahwaz (nur zugewanderte Völker) ausgeschieden. Bei der Beurteilung der Resultate ist aber zu berïcksichtigen, daß wahrscheinlich trotzdem Völker mit einbezogen wurden, deren Ursprung nicht mit dem gegenwärtigen Standort übereinstimmt.

Die p.c.a. des Meda-Clusters - 93 Proben - allein, bzw. gemeinsam mit den beiden morphometrisch am nächsten stehenden Rassen anatoliaca und cypria, zeigt eine sehr deutliche geographische Gliederung innerhalb der Gruppe (Abb. 3). Es sind fünf Cluster zu erkennen, die allerdings nur zum Teil voneinander abgegrenzt sind.

Eine kompakte Gruppe in der Mitte der Graphik (Abb. 3) bilden 35 Proben aus Zentral- und Westiran. Einzelne Proben dieser Herkunft liegen jedoch weit außerhalb des Clusters. Ob dies eine Folge der ausgedehnten Wanderaktivität gerade mit diesen Völkern ist, läßt sich kaum entscheiden.

Völlig getrennt davon liegt ein Cluster mit Proben aus NO Persien (Abb. 3), und weitgehend getrennt die Herkünfte aus SO Anatolien.

Die Proben aus dem Iraq bilden einen keilförmigen Cluster, der in den zentralpersischen eindringt (Faktoren 1 und 2, Abb. 3). Einen Cluster für sich, jedoch mit sehr großer Streuung, bilden die Herkünfte aus SO Iran (Kerman).

Besondere Aufmerksamkeit verdient die morphometrische Einordnung der Proben von der Küstenebene des Kaspischen Meeres. Während alle anderen 
Proben des Meda-Komplexes aus dem Hochland mit klimatischen Extremen stammen, liegt die Küstenebene in einer Depression mit einem feuchten, subtropischen Klima. Der Großteil dieser Proben ist zwar sehr deutlich verschieden von den beiden Nachbarpopulationen des Hochlandes (Zentral- und NO Iran, Tab. 2), sie sind aber nicht zu trennen von den Proben aus Kerman. Obwohl sich also kein eigener Cluster aufstellen läßt, muß man diese Gruppe als das Ergebnis einer eigenständigen Parallelevolution auffassen, mit Konvergenz zur Gruppe Kerman in den analsierten Merkmalen.

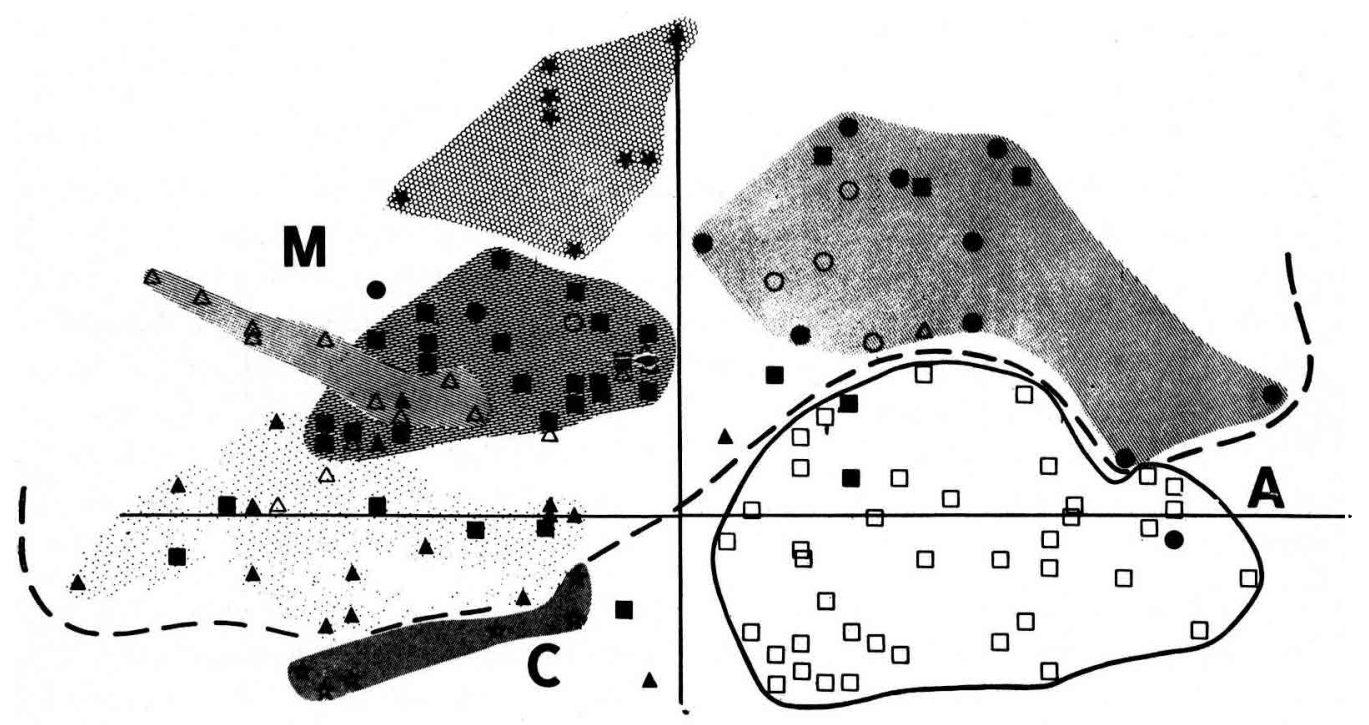
A A.m. anatoliaca
C A.m. cypria
$\square$
M A. m. meda

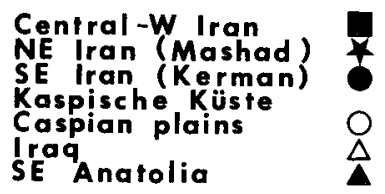

Aвв. 3. - Gesonderte Principal-component-Analyse (Faktor $1+2$ ) von A.m. meda, einschließlich A.m. cypria und A.m. anatoliaca

$$
\mathrm{N}=139, \text { Merkm. }=34
$$

FIG. 3. - Detailed principal component analysis (factor 1 +2 ) of A.m. meda, including A.m. cypria and A.m. anatoliaca

$$
\mathbf{N}=139 \text {, characters }=34
$$


Mit den Daten aus einer schrittweisen Diskriminanzanalyse läßt sich diese morphometrische Gliederung noch übersichtlicher darstellen. Dies ist besonders dann der Fall, wenn man sich mit Konfidenzgrenzen von $75 \%$ begnügt (Abb. 6). (Nach J. HuXLeY (1938) kann man am untersten taxonomischen Rang dann von einer geographischen Gruppierung sprechen, wenn sich $75 \%$ der Individuen dieser Gruppe zuordnen lassen). Die drei Gruppen Z, T und N sind anhand der Faktoren 1 und 2 vollständig voneinander getrennt. Abgesehen von einer kleinen Überlappung zwischen $\mathrm{T}$ und $\mathrm{Z}$ bleibt dies auch bei Konfidenzgrenzen von $90 \%$ (Fig. 4) so. Die Ellipse «I»(Iraq) läßt sich auf einer Darstellung der Faktoren 1 und 3 isolieren (Fig. 5, 6). Der Cluster « $S$ » (Kerman) hingegen zeigt auf allen Darstellungen eine starke Überlappung mit Zentral- und W Iran.

Bei aller geographischen Differenzierung sind die morphometrischen Beziehungen zwischen den einzelnen Gruppen so eng, mit oft sich überlappenden Clustern, daß an der Einheit von A.m. meda als geographischer Rasse nicht gezweifelt werden kann. Diese Populationen werden deshalb als Lokalformen von A.m. meda eingestuft.

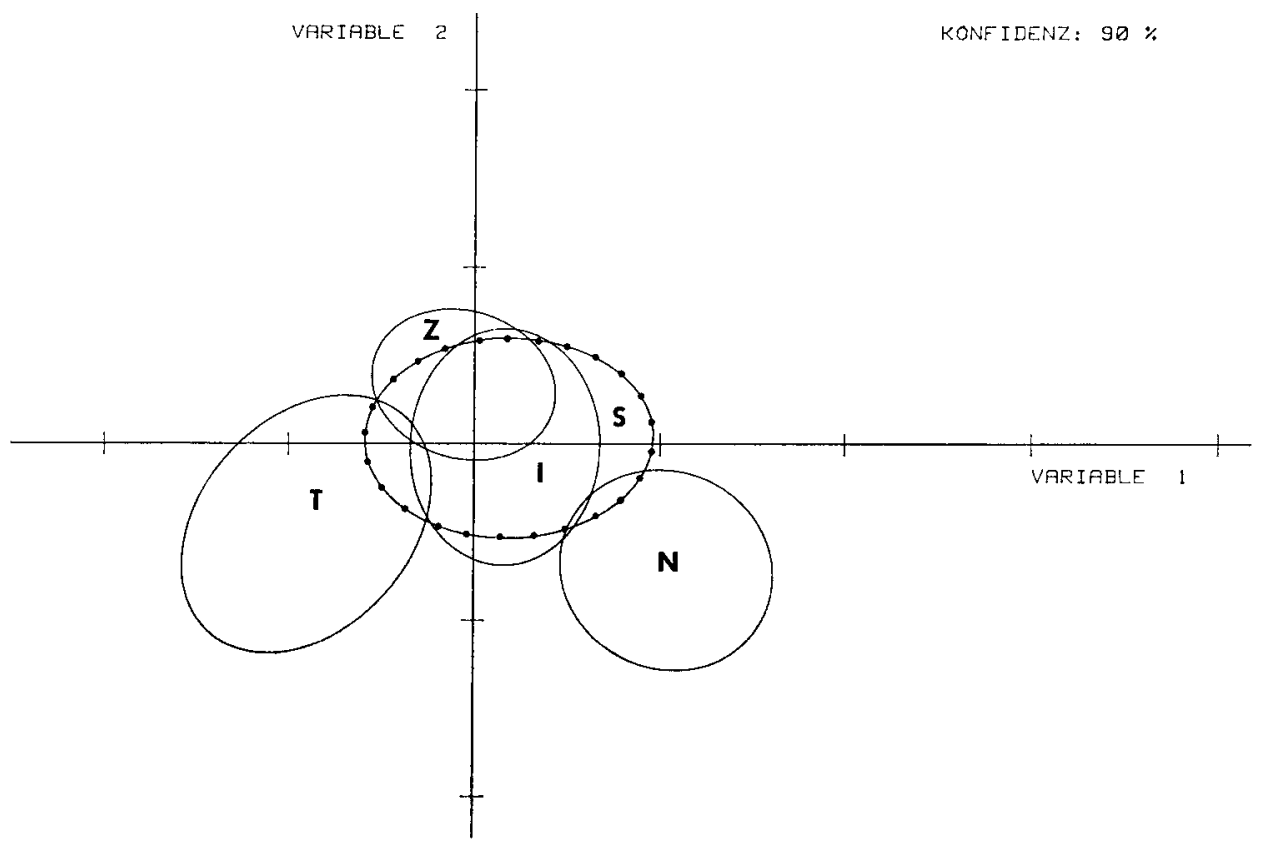

ABB. 4. - Diskriminanzanalyse von A.m. meda

Faktor $1+2$, Vertrauensgrenzen $90 \% . \mathrm{N}=63$, Merkm. $=9 . \mathrm{I}=\mathrm{Iraq}, \mathrm{N}=\mathrm{NO} \operatorname{Iran}$, $\mathrm{S}=\mathrm{SO}$ Iran, $\mathrm{T}=\mathrm{SO}$ Anatolien, $\mathrm{Z}=$ Zentral- und West-Iran

Fig. 4. - Discriminant analysis of A.m. meda

Factor $1+2$, limits of confidence $90 \% . \mathrm{N}=63$, charac. $=9, \mathrm{I}=\operatorname{Iraq}, \mathrm{N}=\mathrm{NE}$ Iran, $\mathrm{S}=\mathrm{SE}$ Iran, $\mathrm{T}=\mathrm{SE}$ Anatolia, $\mathrm{Z}=$ Central and West Iran 


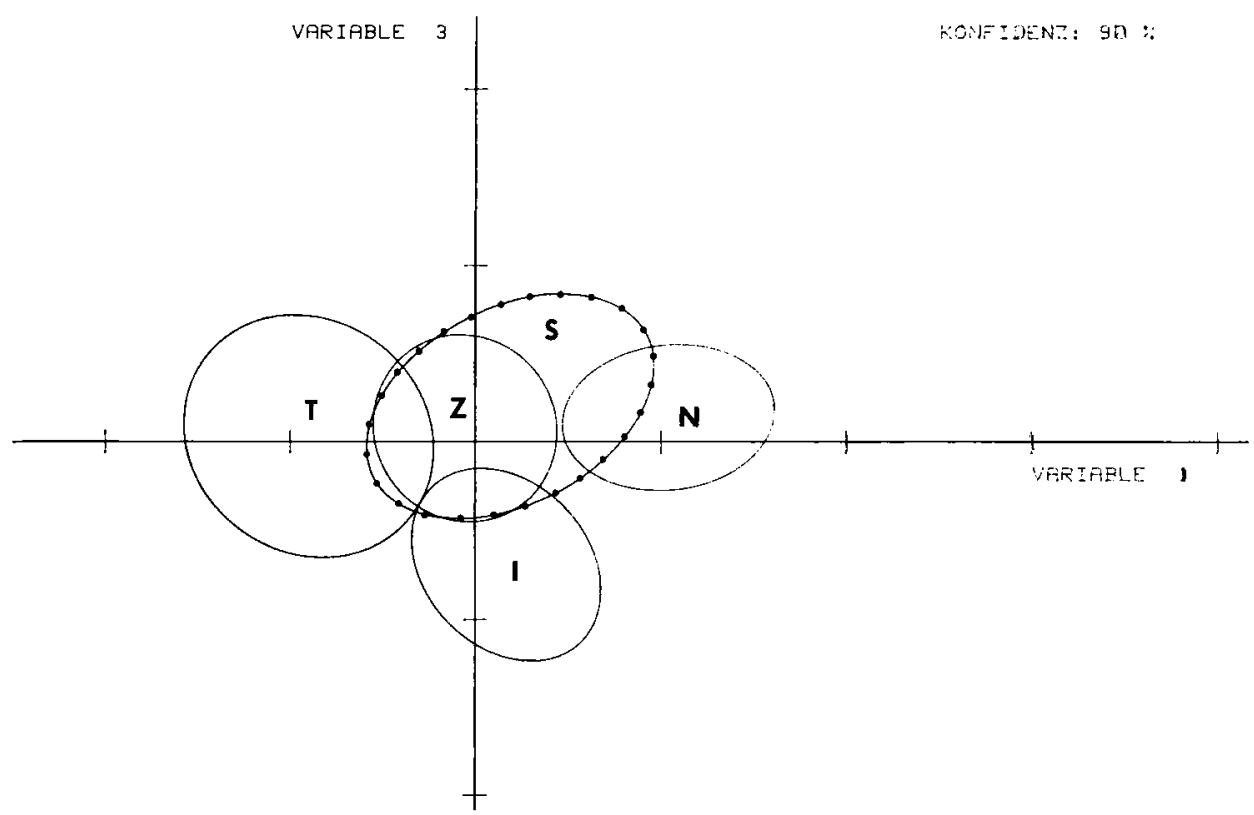

Aвв. 5. - Diskriminanzanalyse ron A.m. meda

Faktor $1+3$, Vertrauensgrenzen $90 \%$ (Abb. 5). Symbole wic in Abb. 4

FIG. 5. - Discriminant analysis of A.m. meda

Factor $1+3$, limits of confidence $90 \%$ (fig. 5). Symbols as in fig. 4

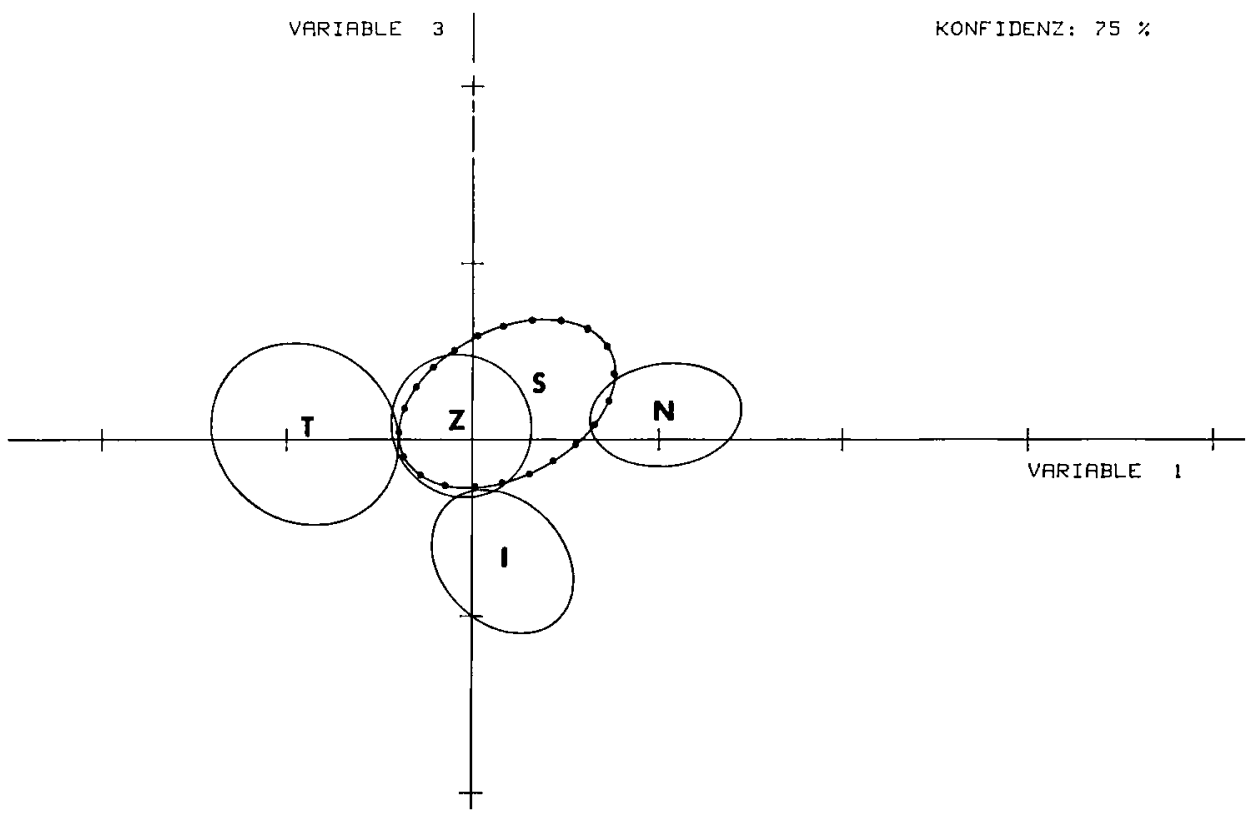

Aвв. 6. - Diskriminanzanalyse von A.m. meda

Faktor $1+3$, Vertrauensgrenzen $75 \%$. Symbole wie in Abb. 4

Fig. 6. - Discriminant analysis of A.m. meda

Factor $1+3$, limits of confidence $75 \%$. Symbols as in Fig. 4 


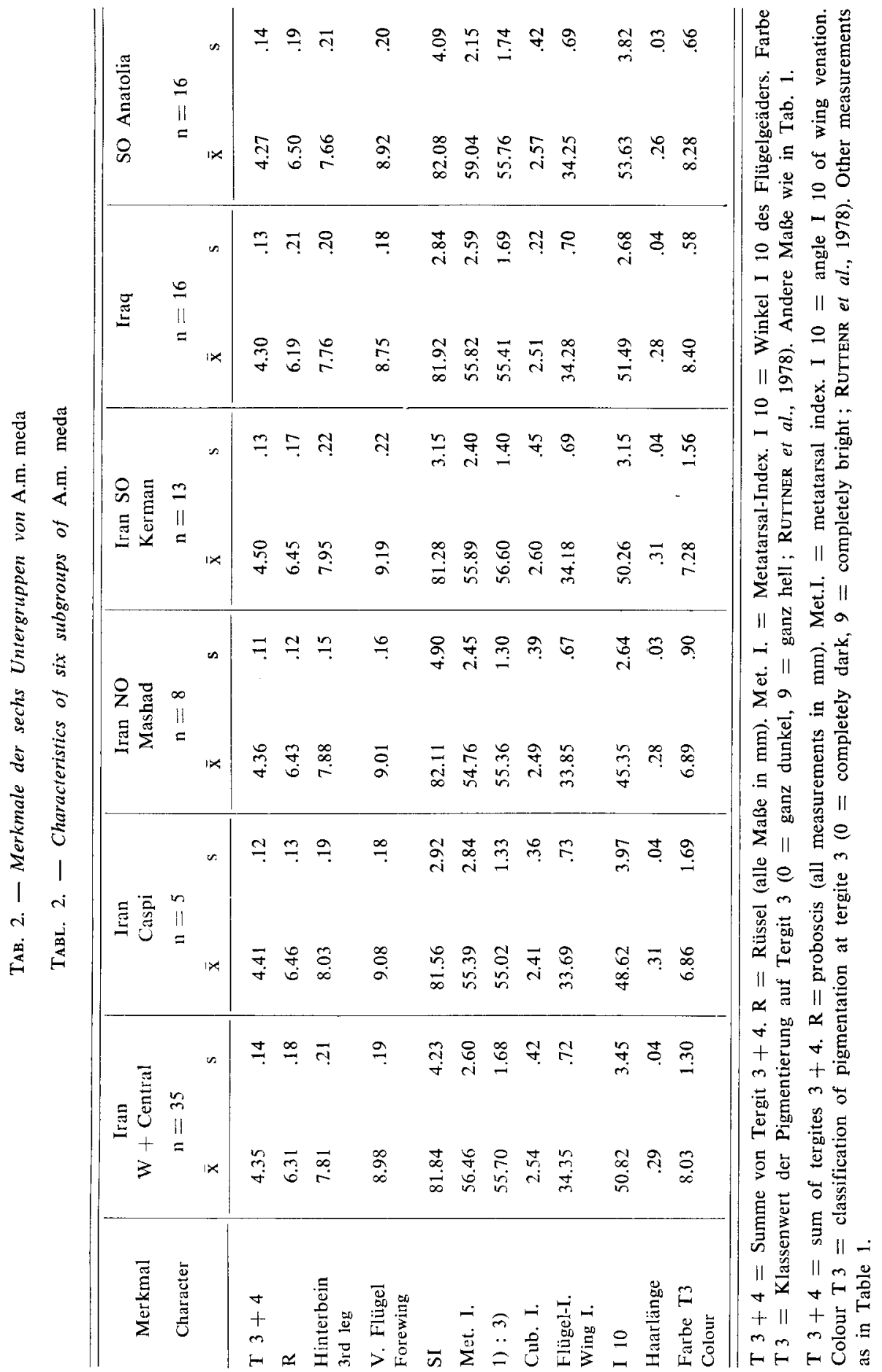


Daß innerhalb von $A . m$. meda morphometrisch charakterisierte Lokalformen differenziert werden konnten, weist darauf hin, daß die Proben überwiegend aus in der Gegend heimischen Völkern entnommen worden sind.

Die Merkmalsunterschiede zwichen den sechs nachstehend beschriebenen Lokalformen (Abb. 7) sind in Tab. 2 zusammengestellt.

1. West- und Zentral-Iran (Lokalform «iranica ») - Diese Form besiedelt das größte Areal (von der iranischen NW Grenze bis nach Estahbanat östlich von Shiras im SO). Sie stellt mit 35 Proben (2/5 des gesamten Materials aus dem Iran) auch den Hauptanteil an den statistischen Daten. Daher kommen die Merkmalswerte dieser Gruppe den Mittelwerten der Rasse sehr nahe. Die Proben stammen aus dem iranischen Hochland (1 200-1 $500 \mathrm{~m}$ ) und aus Orten der Randgebirge bis zu $3000 \mathrm{~m}$ Höhe. Ein wesentlicher Anteil stammt aus den Zentren der Bienenzucht in Azarbeijan und aus dem Kurdischen Bergland.

2. Niederung am Kaspi-See (Massandaran). — Diese Biene ist in fast allen Maßen (einschließlich der Haarlänge) größer als die benachbarten Populationen des um 1200-1 $500 \mathrm{~m}$ höheren Hochlandes; sie hat die längsten Beine und die schmalsten Flügel von allen meda-Formen. Die Pigmentierung ist dunkler, der Cubital-Index (CI) kleiner als bei den anderen Gruppen.

3. Nordost-Iran (Mashad). - Im äußersten NO des mellifera-Areals im Iran befindet sich eine sehr einheitliche, von den anderen Gruppen gut abgegrenzte Form (Abb. 3-6). In den Größenmaßen liegt sie zwischen den Werten von Massandaran und dem zentralen Hochland. Der Grund, daß der Cluster auf Achse 2 nach oben, an die Peripherie des Gesamtclusters verschoben wurde, liegt in den abweichenden Meßdaten für eine Anzahl von Aderwinkeln des Vorderflügels (z.B. I 10 ; RutTNER et al., 1978). Auffällig sind die schmalen Metatarsen von Bein 3. Breite Metatarsen sollen das Kennzeichen der kaukasischen Rassen (remipes $=\ll$ ruderfüßig $»)$ sein.

4. Südost-Iran (Kerman). - Die Biene dieser offensichtlich eng begrenzten Population an der SO Grenze des Mellifera-Areals (zugleich die südlichste Biene des Iran, 29 $\mathrm{N}$ Breite (Abb. 7) ist erstaunlicherweise die größte des Landes. Die Mittelwerte für Körpergröße sowie Flügellänge und -breite sind sogar größer als die der Anatolischen Biene. Bei starker Streuung der Werte erreichen einzelne Proben Rüsellängen von $\bar{x}=6,62 \mathrm{~mm}$ und Flügellängen von $\bar{x}=9,41 \mathrm{~mm}$, womit diese Biene in den Bereich der «großen», nördlicheren Rassen carnica und mellifera vorstößt. Auffällig ist auch der hohe Wert des CI, der in einzelnen Proben einen Mittelwert von fast 3,0 erreicht, sowie die ungewöhnlich große Streuung zwischen der Völkern, die eine Abgrenzung dieser Population von der zentralpersischen Biene unmöglich macht. Möglicherweise befinden sich unter den analysierten Völkern dieses Gebietes neben autochthonen Lokalformen auch zugewanderte Völker. 


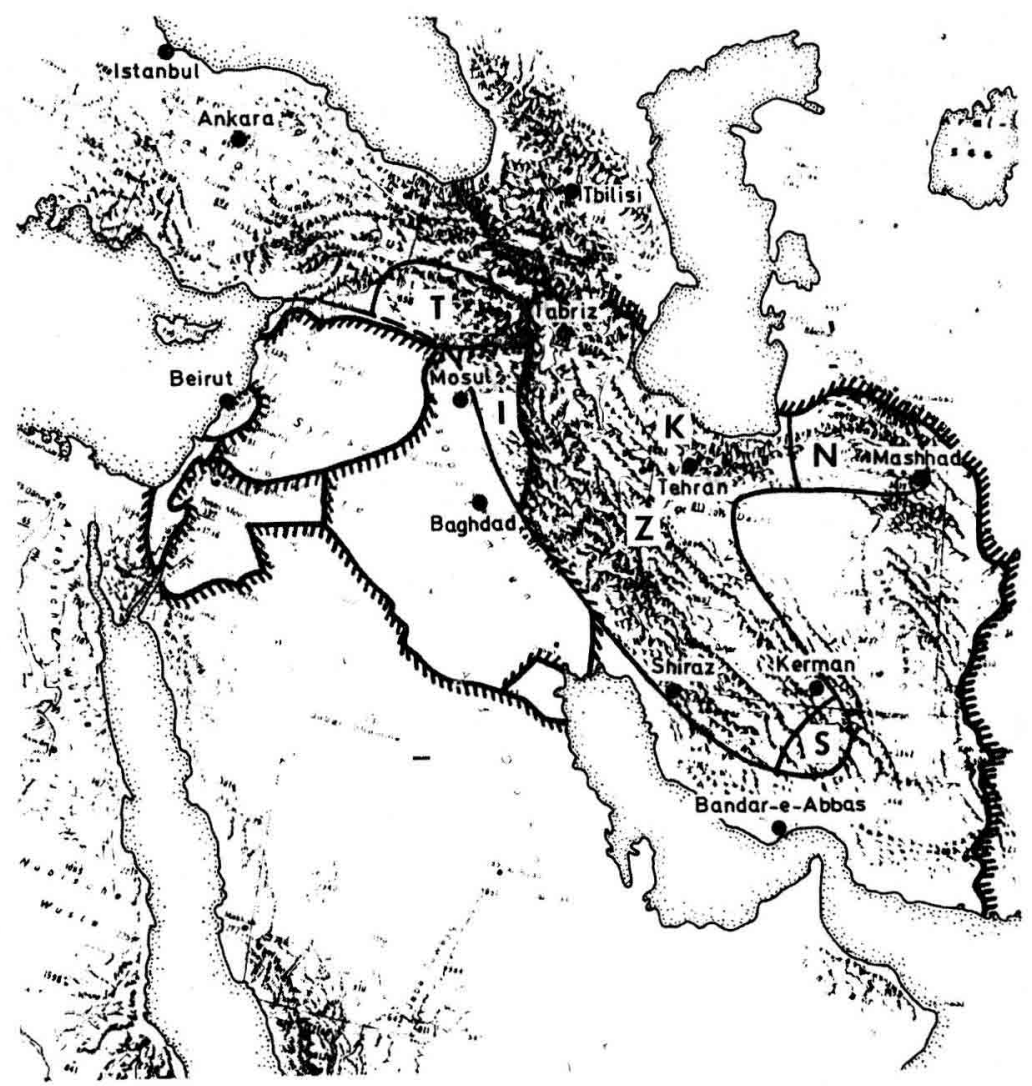

Aвв. 7. - Die Länder des Vorderen Orient mit den Verbreitungsgrenzen ron A.m. meda und ihren Unterpopulationen

Symbole wie in Abb. 4, K = Population am Kaspischen Meer

FIG. 7. - Countries of the Near East with the areas of distribution of A.m. meda and its sub-populations

Symbols as in fig. $4, K=$ population on the Caspian Sea

5. und 6. Die beiden Randpopulationen im SW und W des Meda-Areals, Iraq und SO Anatolien, haben die geringere Körpergröße, hellere Pigmentierung und kurze Behaarung gemeinsam. Ein wichtiger Unterschied zwischen den beiden Populationen ist der kurze Rüssel der Irakischen Biene, während die Südostanatolische zwar das kürzeste Abdomen, aber den längsten Rüssel von allen MedaFormen aufweist. Hervorzuheben ist der breite Metatarsus 3 der SO anatolischen Biene. 


\section{Drohnen}

Von 7 Ständen aus verschiedenen Gebieten des Iran konnten Drohnen untersucht werden (ebenfalls 20 Tiere je Probe). Die Proben stammen aus drei der in dieser Arbeit unterschiedenen Populationen : 1) West- und Zentraliran (Region Urmia-See $2 \mathrm{x}$, Nord-Aserbeijan, Kurdisches Bergland, südlich Teheran je $1 \mathrm{x}$ ) ; 2) Kaspische Küstenebene (Western) ; 3) SO Iran (Kerman).

TAB. 3. - Morphometrische Daten von Drohnen aus dem Iran, im Vergleich zu zwei Proben aus Süd- und SO Europa

TABL. 3. - Morphometric data of drones from Iran, compared with two samples from South and SE Europe

\begin{tabular}{|c|c|c|c|c|c|c|}
\hline \multirow{3}{*}{$\begin{array}{l}\text { Merkmal } \\
\text { Character }\end{array}$} & \multicolumn{2}{|c|}{ meda } & \multicolumn{2}{|c|}{ ligustica } & \multicolumn{2}{|c|}{ carnica } \\
\hline & \multicolumn{2}{|c|}{$\mathrm{n}=140$} & \multicolumn{2}{|c|}{$\mathrm{n}=20$} & \multicolumn{2}{|c|}{$\mathrm{n}=20$} \\
\hline & $\overline{\mathrm{X}}$ & $\mathbf{s}$ & $\overline{\mathbf{x}}$ & $\mathrm{s}$ & $\overline{\mathbf{X}}$ & s \\
\hline $\begin{array}{l}\text { Hinterbein (mm) } \\
\text { Hind leg }\end{array}$ & 9.737 & .131 & 9.833 & 300 & 9.794 & .438 \\
\hline $\begin{array}{l}\text { Vorderflügel (nm) } \\
\text { Forewing }\end{array}$ & 11.941 & .154 & 12.110 & .310 & 12.298 & .389 \\
\hline Cubitalindex & 1.81 & .31 & 2.14 & .22 & 1.85 & .26 \\
\hline $\begin{array}{l}\text { Farbwert Tergit } 2 \\
\text { Colour tergite } 2\end{array}$ & 4.87 & 1.75 & 8.15 & .37 & .25 & .55 \\
\hline
\end{tabular}

Tab. 3 gibt den Vergleich der Mittelwerte aus den 7 Iran-Proben mit Drohnenproben von A.m. ligustica und carnica. Die relativ geringe Streuung der Meda-Daten weist darauf hin, daß sie aus derselben taxonomischen Gruppe stammen. Meda-Drohnen sind im Durchschnitt etwas, aber nicht wesentlich kleiner als Drohnen von ligustica und carnica. Am deutlichsten sind die Unterschiede in der Panzerfärbung: meda ist dunkler als ligustica, aber viel weniger pigmentiert als carnica (ein Farbwert von 4.87 bedeutet, daß am 2. Hinterleibsring breite gelbe Zeichen, aber gerade noch keine Ringe vorhanden sind). Der Cubitalindex liegt im Bereich der Variationsbreite der beiden europäischen Rassen. Wie die Arbeitsbienen, zeigen auch die Drohnen, daß sie morphometrisch den letzteren überraschend nahestehen.

Die Variation innerhalb der Drohnenherkünfte des Iran zeigt ähnliche Tendenzen wie die der Arbeiterinnen: Die Probe Nr. 734 (Region Kerman) und (weniger ausgeprägt) Nr. 780 (Kaspische Küstenebene) sind auch hier die 
größten. Die Kerman-Drohnen erreichen die Größe einer durchschnittlichen carnica-Herkunft (Pr. 207) : Hinterbein $9.992 \mathrm{~mm} \mathrm{(s=0.211)} \mathrm{gegenüber}$ $9.794 \mathrm{~mm}(\mathrm{~s}=0.438)$; Vorderflügel $12.262 \mathrm{~mm}(\mathrm{~s}=0.211)$ gegen $12.298 \mathrm{~mm}$ ( $s=0.389$ ). Mehr noch als die Arbeiterinnen sind die Drohnen aus SO Iran dunkler als der Durchschnitt.

\section{BIOLOGIE VON APIS MELLIFERA MEDA}

Der Großteil der Bienenvölker von A.m. meda wird auch heute noch in Beuten ohne Rähmchen gehalten. Die sich ständig ausweitende Bienenzucht in Magazinen ist noch relativ jung. So ist es verständlich, daß über die physiologischen und Verhaltenseigenschaften dieser Bienen sehr wenig bekannt ist. Die nachstehenden Angaben stammen z.T. aus Erfahrungen, die einer der Autoren (D.P.) in Tabriz sammeln konnte, z.T. von Dipl. Ing. G. Salman aus der Gegend von Mosul. Es ist natürlich sehr gut möglich, wenn nicht wahrscheinlich, daß zwischen den morphologisch unterscheidbaren Typen auch Verhaltensunterschiede bestehen.

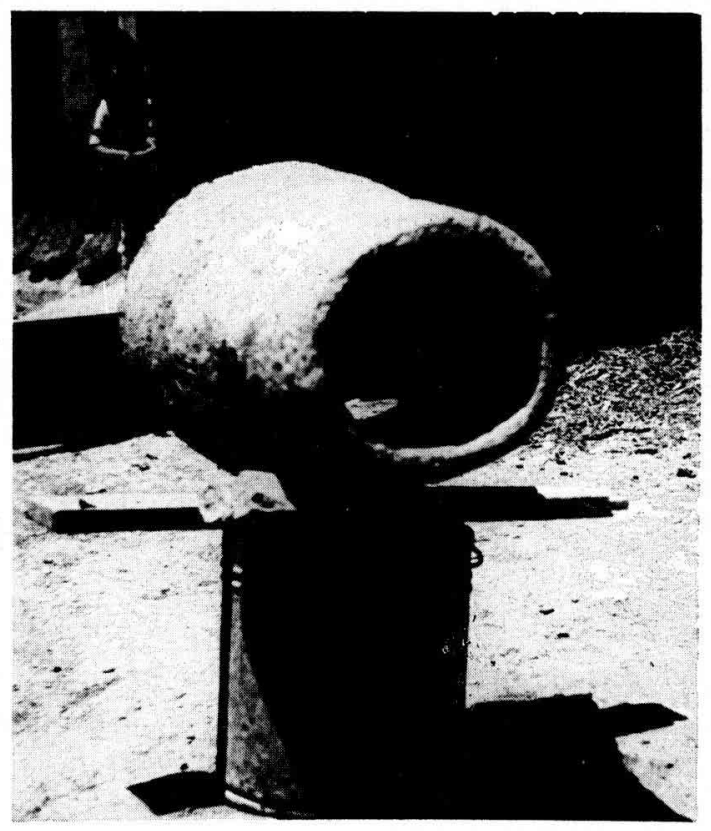

Авв. 8. - Konische Tonröhre als Bienenwohnung

FIG. 8. - Conical clay tube used as bee hive 
Klimatisch ist das ganze Verbreitungsgebiet von A.m. meda ziemlich einheitlich — abgesehen von der feucht-warmen Region am Kaspi-See : Kalte Winter, heisse, trockene Sommer, die in den vier Monaten Juni-September praktisch ohne Niederschlag bleiben. Der Jahresniederschlag überschreitet nur im Gebirge $1000 \mathrm{~mm}$, im Süden bleibt er an vielen Orten unter $300 \mathrm{~mm}$. Um ein Beispiel herauszugreifen : Am Fundort Kumrang im Zagros-Gebirge (Pr. Nr. 721), $2650 \mathrm{~m}$ ü. NN, beträgt der Jahresniederschlag $1270 \mathrm{~mm}$. Fröste sind in sechs Monaten des Jahres zu erwarten, das mittlere Januarminimum beträgt $-14,7^{\circ}$, das mittlere Maximum im Juli $39^{\circ}$. Weiter im Süden überschreiten die Sommertemperaturen im Hochland $40^{\circ}$, in der Küstenebene am Persischen Golf $50^{\circ}$.

Die an diesen Klimatyp angepaßte Biene muß die Fähigkeit einer längeren Winterruhe besitzen. Rückschlüsse auf das ökologische Anpassungsspektrum der Persischen Biene erlauben die Erfahrungen mit importierten Italienischen Bienen, die sich in diesem Lande sehr gut halten. Die Ähnlichkeiten zwischen diesen beiden Rassen beschränken sich offensichtlich nicht auf die Morphologie.

Die Schwarmtendenz von A.m. meda ist i.a. hoch. Übereinstimmend wird angegeben, daß es auch bei reichlichem Raumangebot in Magazinkästen zu Schwärmen kommt. Die Zahl der Weiselzellen im Schwarmvolk wird sowohl für den Iran wie für den Iraq mit 10-20 angegeben. Dies steht in Widerspruch zu Angaben SkoRIKows, der von hunderten Schwarmzellen spricht, ähnlich wie bei anderen orientalischen Rassen. Völiges Ausziehen von Völkern (absconding) oder Wanderschwärme wurden nicht beobachtet. Im Süden beginnt die Schwarmzeit schon im März, in Azerbeijan liegt sie in den Monaten Juni-Juli. Der Brutrhythmus der Völker ist den klimatischen Bedingungen angepaßt. Im Norden gibt es eine winterliche Brutpause, im Süden nicht. Drohnenbrut erscheint im Süden im März, im Norden im Mai.

Kittharz wird reichlich verwendet. Bei manchen Völkern wird im Winter das Flugloch mit Propolis eingeengt.

Die Verteidigungstendenz der Bienen scheint im Iraq größer zu sein als in Azarbaijan : Die Bienen in Mosul verfolgen den Imker nach einem Eingriff über eine Distanz $200 \mathrm{~m}$ gegenüber $30 \mathrm{~m}$ in Tabriz. Dort könne man an den Völkern ohne Handschuhe arbeiten, was im Süden nicht möglich sei. Doch gibt es in dieser Hinsicht offensichtlich eine erhebliche Variabilität : Die kurdischen Imker unterscheiden einen sanften Stamm (Bezin $=$ «Schafe ») und einen aggressiven (Mirr $=\ll$ Ziegen $» ;$ KHALEL, 1955).

Eine interessante Beobachtung wird aus dem Iraq mitgeteilt: Die Bienen reagieren auf die Rufe (oder optischen Signale ?) der in Schwärmen angreifenden Merops (Merops) durch sofortiges Einstellen des Fluges. 
Im Iran gibt es in der traditionellen Imkerei eine Vielfalt von Beutentypen : Liegende und stehende Klotzbeuten, geflochtene Walzen, Tongefäße verschiedener Form (Abb. 5). Im Iraq werden Bienenvölker in Tonröhren gehalten, die zu 30 oder mehr gestapelt sind, entsprechend der alten Tradition ostmediterraner Kulturen. Die Honigentnahme erfolgt von rückwärts. Die Magazinimkerei hat sich vor allem im Umkreis der großen Städte entwickelt. Unter Ausnützung der Trachtunterschiede wandern die Berufsimker des Azarbaijan mit hunderten von Völkern im Sommer ins Gebirge oder in spezielle Trachten. Dabei kommt es zu großen Ansammlungen von Bienenvölkern (Abb. 9).

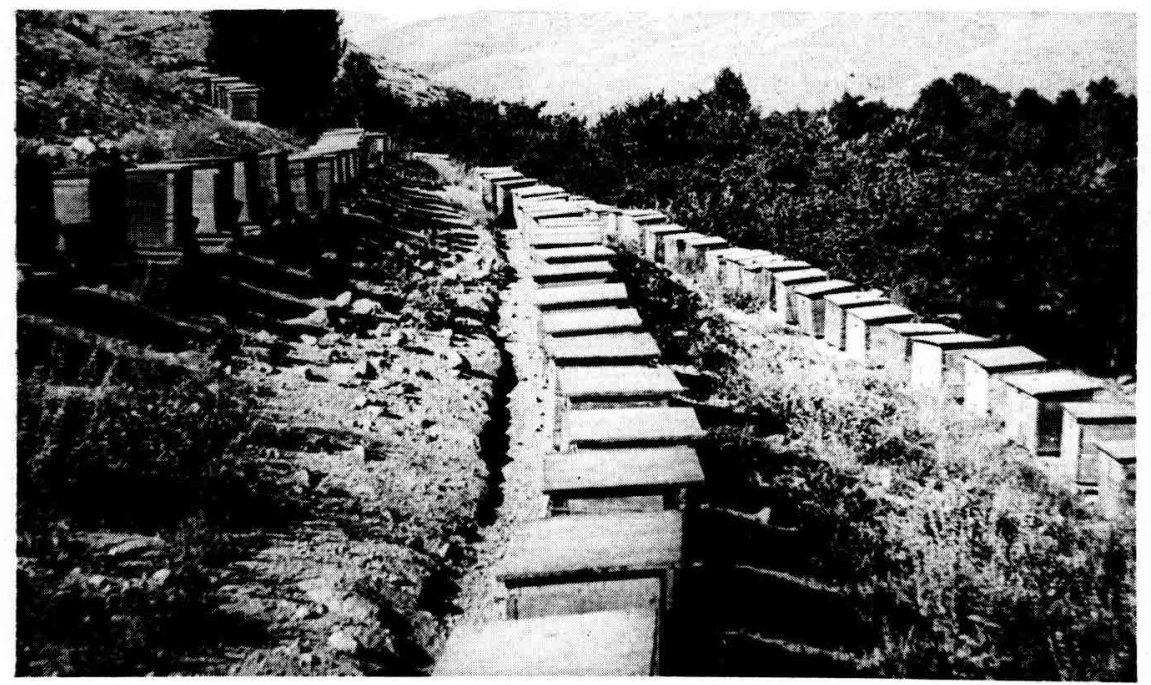

Авв. 9. - Wanderstand in Azarbeijan mit Langstroth-Kästen

FIG. 9. - Migratory apiary in Azarbeidjan with Langstroth hives

\section{DISKUSSION}

Apis mellifera meda SKoRIKow ist eine geographische Varietät, die sich von allen bisher von uns morphometrisch analysierten Rassen klar abgrenzen läßt. Die Beziehungen zur nördlich anschließenden Armenischen Biene sind noch nicht geklärt. SKorikow (1929) hebt die Unterschiede zwischen diesen beiden Rassen hervor, ohne sie allerdings im einzelnen zu spezifizieren.

Meda ist das östlichste Glied der geographischen Rassen von Apis mellifera. Man muß die Frage stellen, warum das Verbreitungsareal dieser Art hier seine 
Grenze findet. $A$. florea weist ein vermutlich ununterbrochenes Areal von Indien nach Westen bis in das Zweistromland auf. Wie WhITcombe in Oman zeigen konnte, erträgt $A$. florea offenbar extrem hohe Temperaturen eher als $A$. mellifera. Die äußersten Fundstellen von $A$. mellifera im SO des Iran befinden sich im östlichsten Abschnitt des südlichen Randgebirges (Abb. 7). Die Hochflächen liegen hier um $2000 \mathrm{~m}$, die Gipfel erreichen mehr als $4000 \mathrm{~m}$. Dementsprechend steigen die mittleren Temperaturmaxima im Sommer nicht über $40^{\circ}$. Weiter im SO hingegen lagen diese Maxima von allen Orten, von denen wir über meteorologische Daten verfügen (Bam, Jiruft, Manujan, Iranshar, Karvandar) durchwegs zwischen $45-52^{\circ}$.

An der nordöstlichen Arealgrenze von A. mellifera enden im Raume Mashad die Ausläufer des Alborz-Gebirges und des Kopetdag. Die Steppe östlich davon ist kein Biotop für Bienen. In die Orte von Turkmenien wurden erst im 19. Jhdt. Honigbienen gebracht (Alpatow, 1948). Hier im Norden beträgt die bienenfreie Distanz zum westlichsten Vorkommen von $A$. cerana FABR. nur $500 \mathrm{~km}$. Sowohl im $\mathrm{N}$ wie im $\mathrm{S}$ liegt ein für beide Arten nicht bewohnbares Gebiet.

Die beiden Arten $A$. mellifera und $A$. cerana stehen einander in vielen Merkmalen sehr nahe. Nach der Stufe der Artdifferenzierung, auf der sie sich befinden, muß man annehmen, daß es sich um junge Arten handelt (RutTNER und MAUL, 1983). Es kann vermutet werden, daß ihre Trennung durch geographische Isolation im Ostiran erfolgt ist. Die iranischen Wüsten waren schon im Tertiär (Oligocaen) vorhanden. Während einer Klimaänderung könnte eine Waldverbindung zu den iranischen Randgebirgen bestanden haben, durch die eine Apis-Art vom Indischen Subkontinent nach Westen vorgedrungen ist. Mit der Unterbrechung dieser Verbindung war die Möglichkeit zur Evolution der beiden Populationen zu eigenen Arten gegeben.

Trotz dieser Beziehungen wurden weder bei den westlichsten Vertretern von $A$. cerana in Afghanistan und Pakistan (eigene unveröffentlichte Daten) noch bei A.m. meda irgendwelche intermediäre Typen gefunden, wie dies für den Kashmir behauptet worden ist (Shah 1980). Bei je zwei Völkern im NO (Mashad) und SO (Kerman) wurde am Hinterflügel nach der artspezifischen "Cerana-Ader » gesucht. Alle Bienen zeigten den reinen Mellifera-Typ.

Die Hypothese, daß A.m. meda historisch mit der Entstehung von A. mellifera verknüpft ist, wird gestützt durch die Feststellung, daß sie gemeinsam mit drei anderen Rassetypen anatoliaca, syriaca und sahariensis in der morphometrischen Struktur von A. mellifera, wie sie sich bei graphischer Darstellung der Resultate einer Mulivariat-Analyse darstellt, eine zentrale Position einnimmt (RUTTNER et al., 1978). Weitere Studien an den Bienen dieser Region sind notwendig, um diese Zusammenhänge genauer zu belegen.

Es überrascht, daß die Grenze von meda in Anatolien sehr scharf gezogen 
werden kann, obwohl hier geographische Barrieren und wesentliche klimatische Unterschiede fehlen. Die Cluster «SO Anatolien» (A.m. meda) und «West- und Zentralanatolien $\gg($ A.m. anatolica) liegen voneinander getrennt, die Bienen beider Formen sind durch beträchtliche Merkmalsunterschiede charakterisiert (Abb. 4, Tab. 1).

Ungeklärt it die Frage, ob es im SW Kontakte zur Syrischen Biene gibt. Abdellatif et al. (1977) zählen die von ihnen aus der Zentralregion des Iraq untersuchten Bienen zu A.m. syriaca. Dazu stehen unsere Befunde in Widerspruch. Allerdings zeigen die von den genannten Autoren mitgeteilten Werte eine so extrem hohe Streuung daß ein Vergleich nicht möglich ist (Rüssellänge 4.3-7.0, $\overline{\mathrm{x}}=5,3 \mathrm{~mm}$; Vorderflügel 7.5-9.6, $\overline{\mathrm{x}}=8,8 \mathrm{~mm}$ ). Bisher ist es uns noch nicht gelungen, Bienen aus Syrien zu untersuchen.

Iran mit seinen großen Gegensätzen zwischen dem extrem kontinentalen Klima des Hochlandes und dem subtropischen Klima am Kaspi-See und am Persischen Golf bietet die Möglichkeit, die adaptive Bedeutung von morphometrischen Merkmalsunterschieden zu untersuchen. An der ganzen westafrikanischen Küste, und weiter nach Norden bis nach Skandinavien läßt sich em enger Zusammenhang zwischen geographischer Breite und Körpermerkmalen nachweisen : In Richtung zum Äquator (und zwar sowohl von Norden wie von Süden) werden die Bienen kleiner und kurzhaariger (RutTNER, 1985). Das entspricht der bekannten Bergmannschen Regel bei Vertebraten. Derselbe Trend der Variation ist in Ostafrika mit zunehmender Meereshöhe festzustellen, wiederum analog zu häufig beobachteten Variationsreihen bei Wirbeltieren: Bergformen sind größer und langhaariger, Formen des Tieflandes sind kleiner und kurzhaariger. Betrachtet man die Variation innerhalb der Meda-Gruppe, so lassen sich nicht die geringsten Anzeichen einer ähnlichen Tendenz feststellen. Im Gegenteil - die Bienen von der Küste des in einer Depression gelegenen Kaspi-Sees (NN-27 m) sind deutlich größer als die des um 1200-2 500 m höher gelegenen zentraliranischen Berglandes. Die größten (und auch rel. langhaarigsten) Formen wurden im SO gefunden (Tab. 2), am Rande der Ebene von Sabzevaran, die wegen ihres milden Winterklimas (Fröste treten kaum auf) zur Obst- und Gemüsekammer für den Norden geworden ist. Wäre die Bergmannsche Regel auch hier gültig, dann müßten die Bienen vom Kaspi-See und vom warmen SO kleiner sein als die des Hochlandes.

Die beiden westlichsten Untergruppen von A.m. meda, die Bienen aus dem Iraq und aus SO Anatolien, sind von rel. kleiner Körpergestalt (Tab. 2). Aber nur die Proben aus dem Iraq stammen aus geschützten, nach Süden offenen Tälern und aus geringerer Meereshöhe. Nur diese eine Gruppe paßt also in das Schema der Bergmannschen Regel. Das Meda-Areal in SO Anatolien hingegen, mit einer ebenso kleinen Biene, unterscheidet sich weder in Höhenlage noch im Klima sehr wesentlich vom Iranischen Hochland. 
Schon bei der Diskussion der «Geokline» an der Atlantikküste wurde darauf hingewiesen, daß es auch bei der Biene Ausnahmen von dieser zoogeographischen Regel gibt (RUTTNER, 1985). Das Beispiel der geographischen Variabilität von A.m. meda zeigt erneut, daß Befunde auch dann nicht ohne weiteres verallgemeinert werden dürfen, wenn sie mit anerkannten Regeln der Zoogeographie übereinstimmen.

Eingegangen im April 1985. Angenommen im Juni 1985.

\section{DANKSAGUNG}

Die Deutsche Forschungsgemeinschaft hat diese Untersuchung durch eine langjährige Beihilfe gefördert. Eine wichtige Unterstützung hat diese Arbeit durch das Forschungsministerium der Islamischen Republik Iran erfahren, für die aufrichtig gedankt wird.

\section{RESUME \\ LES ABEILLES D'IRAN. \\ 2. APIS MELLIFICA MEDA SKORIKOW, L'ABEILLE PERSE}

Apis mellifica est présente dans toute la partie montagneuse de l'Iran, là où croît une végétation suffisante. A.S. SкоRıкоw a caractérisé en 1929 une nouvelle race d'abeille, Apis mellifica meda, dans la région frontalière entre l'Union soviétique et l'Iran, mais jusqu'à présent aucune analyse morphométrique n'avait été réalisée.

63 échantillons, de 20 abeilles chacun, ont été prélevés dans les diverses régions de I'Iran et analysés selon une méthode déjà décrite. A titre de comparaison on a prélevé dans les pays voisins et en Italie 142 échantillons : Turquie 63, Irak 16, Liban-Israël-Jordanie 21, Chypre 6 et Italie 36. L'analyse statistique a été faite selon diverses méthodes d'analyse multivariable (en composante principale et discriminante).

La plus forte densité d'abeilles (et le centre de l'activité apicole professionnelle) se trouve dans le nord-ouest de la Perse, en Azerbaïdjan. Vers l'est, on trouve au nord, des colonies d'abeilles jusque dans la région de Mashad et au sud, jusque dans la région de Sabzewaran, au sud-est le Kernan. Cela correspond à la partie montagneuse de l'Iran. Les déserts de Kavir et de Lut en Perse centrale, dépourvus d'abeilles, donnent à l'aire d'Apis mellifica en Iran une forme de langue ouverte à l'est (Fig. 1). La distance à la limite occidentale, connue à ce jour, d'Apis cerana en Afghanistan et Pakistan est supérieure à $500 \mathrm{~km}$. Jusqu'à présent Apis cerana n'a pas été trouvée en Iran, pas plus que des formes de passage. Avec $A$. florea sur le bord du Golfe Persique il y a des contacts intermittents, mais aucune présence sympatrique durable. Le facteur d'isolement écologique entre les deux espèces est visiblement une température estivale supérieure à $45^{\circ} \mathrm{C}$.

Les analyses multivariables ont fourni un nuage fermé pour tous les échantillons provenant d'Iran. A ce nuage appartiennent également tous les échantillons des zones montagneuses d'Irak et un groupe provenant du sud-est de l'Anatolie (Fig. 2). Cette entité taxonomique est nommée A.m. meda Skorikow. Les nuages d'Anatolic centrale et occidentale, ainsi que ceux d'A.m. cypria et $A . m$. syriaca sont nettement séparés.

A.m. meda ressemble fortement à A.m. ligustica (Tabl. 1), qui s'en distingue néanmoins sur le plan morphométrique et comportemental. Les mâles ne sont qu'un peu plus petits que ceux de ligustica, mais nettement plus foncés (Tabl. 3). Les caractéristiques éthologiques d'A.m. meda ne sont pas totalement connues. Sa tendance à l'essaimage est moyenne et forte, de même que sa propension à la défense. Le nombre de cellules royales d'essaimage n'est pas supérieur à 10-20. Elle propolise beaucoup. 
A l'intérieur d'A.m. meda même, il y a aussi une différenciation morphométrico-géographique. Les colonies de Perse centrale et occidentale constituent le groupe le plus important (Fig. 3-6). Se différencient de ce groupe les abeilles des régions basses de la Mer Caspienne et celles du nord-est et sud-est de la région frontalière de l'aire de mellifica. Les abeilles d'Irak et celles du sud-est de l'Anatolie forment également un groupe propre, si bien qu'on peut définir 6 sousgroupes au sein d'A.m. meda (Tabl. 2). Les 2 groupes « Mer Caspienne» et «Sud-Est» géographiquement très éloignés se recouvrent l'un l'autre sur la représentation graphique (Fig. 4).

La variabilité au sein de l'abeille perse ne montre aucunes tendances qui puissent s'accorder avec la règle de Bergmann (formes plus grosses et à pilosité plus longue dans le nord ou dans les zones d'altitude). C'est au contraire les abeilles du sud, ou des zones basses, qui sont plus grosses que celles des montagnes du nord.

La ceinture désertique de l'Iran, restée pour l'essentiel inchangée depuis l'Oligocène, pourrait être à l'origine de la séparation du couple cerana-mellifica. On peut supposer qu'une population d'Apis, venant du sous-continent indien, ait réussi à un moment donné à pénétrer vers l'ouest au nord du Kavir. Selon cette hypothèse un isolement total, et par conséquent la possibilité pour une espèce de se former, aurait pu se produire après interruption de la liaison (qui devait être nécessairement restreinte).

\section{SUMMARY}

THE HONEYBEES OF IRAN.

\section{APIS MELLIFERA MEDA SKORIKOW}

Apis mellifera meda is found in all the highlands of Iran, provided sufficient vegetation is present. In 1929, A.S. Skorikow named a race of bees of the Sowjet-Iranian border region «A.m. meda». So far no morphometric analysis of the honeybees of Iran has been made. Sixty three samples of 20 bees each from all parts of Iran were collected. One hundred forty two samples from neighboring countries (Turkey 63 samples, Iraq 16, Lebanon, Israel and Jordan 21, Cyprus 6 and from Italy 36) were included for comparison. Measurements of several characters were made according to methods described earlier. For statistical analysis different multivariate methods (principal component and discriminant analysis) were applied.

The highest bee density (the center of professional apiculture) exists in NW lran (Azarbaijan). In the east, honeybee colonies were found as far as the area of Mashad in the north, and as far as Sabzevaran (SE of Kerman) in the south. This corresponds to the area of the Iranian highland. Since the deserts of Central Persia, Kavir and Lut, are void of bees, the area of $A$. mellifera has the shape of a clamp, open to the east (Fig. 1). The distance to the area of $A$. cerana in Afghanistan and Pakistan exceeds $500 \mathrm{~km}$. Neither $A$. cerana nor intermediates between it and A. mellifera have been found in Iran. Temporary contacts, but no permanent coexistence occur with $A$. florea at the Persian Gulf. The isolating factor between the two species seems to be an annual temperature maximum higher than $45^{\circ}$.

The multivariate analysis of the data resulted in a compact cluster of all the samples of Iran, including also all the samples from the mountain region of Iraq and of a restricted area in SE Anatolia (Fig. 2). This taxonomic unit is called A.m. meda Skorikow. Distinctly separated clusters were found for the bees of Central and West Anatolia, for A.m. cypria and for A.m. syriaca.

A.m. meda is very similar to A.m. ligustica, through discriminated by several morphological and behavioral characters (Tabl. 1). The drones are only slightly smaller than ligustica drones, but distinctly darker (Tabl. 3). The behaviour of A.m. meda is known only in part : the swarming tendency is medium to high, and so is the defense reaction. The number of queen cells during the swarming season rarely exceeds 10-20. Ample propolis is used.

A morphometrical-geographic differentiation is also found within A.m. meda. The largest group - as to number of samples as well as to area - consists of the colonies of Central and West Iran (Fig. 3-6). Distinct groups are found in the lowlands of the Caspian Sea and in 
the northeastern and southeastern border regions of the meda area in Iran. The bees of Iraq and southern Anatolia constitute morphometrically separate groups also. Thus six subgroups can be discriminated within $A . m$. meda (Tabl. 2, although two of them, the geographically far distant «Caspian Sea» and «southeastern» groups overlap on the graph (Fig. 4).

Variability within A.m. meda shows no tendency consistent with Bergmanns rule (larger and long-haired bees in the north and in higher altitudes). On the contrary, in Iran the bees of the south and of the lowlands are larger in general than those of the northern highlands.

The desert belt of Iran, basically unchanged since the oligocene, could have been the prerequisite for the separation of the sister species cerana-mellifera. Supposing an advance to the west, north of the Kavir, of an Apis population of the Indian subcontinent, the connection with the main area would necessarily have been very narrow. An interruption of this connection by a climatic change could have resulted in a complete isolation, creating the possibility of the origin of separate species.

\section{BIBLIOGRAPHIE}

Abdellatif A.M., Abu-Elnaga A.M., Ali M.H., Shakir P.M., Jalili K.K., 1977. - Biometrical studies on Iraqi honeybees. J. apic. Res., 16, 143-144.

Adam Bruder, 1983. - Auf der Suche nach den besten Bienenstämmen. Delta Verlag, St.Augustin.

Alpatow W.W., 1948. - [The strains of the honeybee and their utilization in agriculture.] Sredi prirodi, vol. 4, Soc. Study of Natur Moskwa (Russ.).

EhLERs E., 1980. - Iran. - Grundzüge einer geographischen Landeskunde. Wissenschaftl. Verl. Ges. Darmstadt.

Khalel Jasim, 1955. - L'apiculture en Irak. La Gazette Apicole, 1955, 10-12.

KLOFT W. u. E., 1971. - Bienenfunde in Nuristan und im Südkaspischen Tieflandwald. Allg. dtsch. Imkerztg., 5 (2), 30-32.

NoGge P., 1974. - Die geographische Verbreitungsgrenze zwischen Westlicher und Östlicher Honigbiene. Allg. dtsch. Imkerztg., 8 (2), 163-165.

Pourasghar D., 1979. - Bienenhaltung im Iran. Verh. 27. Int. Bienenzucht Kongr., Apimondia Verl., Bukarest, 321-323.

Ruttner F., 1975. - Die Bienenrassen Afrikas. Verh. 25. Int. Bienenzucht Kongr., Apimondia Verl., Bukarest, 344-364.

Ruttner F., 1985. - Graded geographic variation in honeybees. Pszczel. Zesz. Nauk (in press).

RuttNer F., Maul V., 1983. - Über die Kreuzungsbarriere zwischen A. mellifera und $A$. cerana. Apidologie, 14, 309-327.

Ruttner F., Pourasghar D., Kauhausen D., 1985. - Die Honigbienen des Iran. 1. Apis florea Fabr. Apidologie, 16, 119-138.

Rutiner F., Tassencourt L., Louveaux J., 1978. - Biometrical-statistical analysis of the geographic variability of Apis mellifera. Apidologie, 9, 363-381.

Schneider P., Djallal A.S., 1970. - Vorkommen und Haltung der Östlichen Honigbiene (Apis cerana FABR.) in Afghanistan. Apidologie, 1, 329-341.

Sнah A.M., 1980. - Beekeeping in Kashmir. 2nd Int. Conference Apic. Trop. Climate, Indian Agric Res. Inst. New Delhi, 197-204.

SkoRIKow A.S., 1929. - [Beiträge zur Kenntnis der kaukasischen Honigbienenrassen.] Rep. appl. Entomol., 4, 1-59. Leningrad (Russ. mit deutscher Zusammenfassung).

Tirgari S., 1971. - Biologie und Verhaltenscharakteristika der iranischen Zwergbiene (Apis florea). Verh. 23. Int. Bienenzucht Kongr., Apimondia Verl., Bukarest, 344-345.

Whitcombe R., 1984. - The Bedouin bee. Aramco World News, 35 (21), 34-41. 